\title{
Ventricular tachycardia triggered by pregnancy in left-ventricular non-compaction cardiomyopathy: a controversial indication to automated defibrillator implantation
}

\author{
Elisa Gherbesi, ${ }^{1}$ Carla Bonanomi, ${ }^{2}$ Vera Bottari, ${ }^{3}$ Gian Battista Danzi ${ }^{4}$ \\ ${ }^{1}$ University of Milan, Milan; ${ }^{2}$ Department of Cardiology, Fondazione IRCCS Cà Granda Ospedale Maggiore \\ Policlinico Milano; ${ }^{3}$ Department of Cardiology, Ospedale Santa Corona, Pietra Ligure; ${ }^{4}$ Department of Cardiology, \\ Ospedale di Cremona, Cremona, Italy
}

\begin{abstract}
Left-Ventricular Non-Compaction (LVNC) is a rare form of cardiomyopathy. Its clinical presentation is highly variable and during pregnancy it is frequently associated with heart failure, embolic events, and arrhythmias. Herein we report a case of a
\end{abstract}

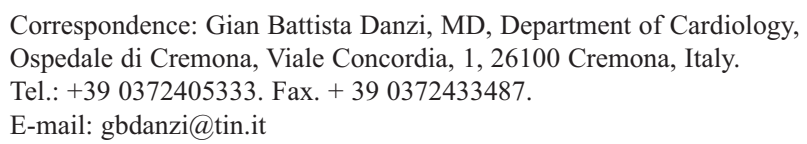

Key words: Isolated Noncompaction of the Ventricular Myocardium; Cardiomyopathies; Cardiac arrhythmias; Pregnancy.

Contributions: All the authors contributed to the conception and design of the article, drafted the article, then revised it, and gave the final approval of the version to be published. All the authors are in agreement to be accountable for all aspects of the work.

Conflict of interest: the authors have no conflict of interest to declare.

Availability of data and materials: All data underlying the findings are fully available.

Ethics approval and consent to participate: No ethical committee approval was required for this case report by the Department, because this article does not contain any studies with human participants or animals. Informed consent was obtained from the patient included in this study.

Consent for publication: The patient gave her written consent to use her personal data for the publication of this case report and any accompanying images.

Received for publication: 31 May 2020.

Accepted for publication: 19 June 2020.

${ }^{\circ}$ Copyright: the Author(s), 2020

Licensee PAGEPress, Italy

Monaldi Archives for Chest Disease 2020; 90:1280

doi: 10.4081/monaldi.2020.1280

This article is distributed under the terms of the Creative Commons Attribution Noncommercial License (by-nc 4.0) which permits any noncommercial use, distribution, and reproduction in any medium, provided the original author(s) and source are credited. woman with LVNC who had an automated defibrillator implantation for recurrent ventricular arrhythmias during pregnancy. During pregnancy and at long-term follow-up no interventions of the device were documented. In conclusion, the management of malignant arrhythmias during pregnancy is one of the concerns for patients with LVNC and requires a careful approach in third-level centers.

\section{Introduction}

Left Ventricular Non-Compaction (LVNC) is a structural abnormality of the ventricular myocardium of uncertain etiology that is considered as primary genetic cardiomyopathy by the American Heart Association, and as unclassified cardiomyopathy by the European Society of Cardiology $[1,2]$. Subjects with LVNC are most commonly asymptomatic; about two-thirds of these patients develop heart failure due to ventricular dysfunction, whereas embolic events, arrhythmias and sudden cardiac death are less frequent. Little is known about the effects of pregnancy on LVNC, nor the safety of pregnancy in patients with LVNC. Pregnancy is characterized by major physiological changes that could lead to hemodynamic compromise and heart failure; in particular, given the specific arrhythmogenic predisposition of this cardiomyopathy, the same hemodynamic load can also determine an arrhythmic risk. We describe the case of a pregnant woman with LVNC who presented multiple episodes of non-sustained ventricular tachycardia (NSVT) and was treated with automated defibrillator implantation.

\section{Case report}

A 43-year-old pregnant woman who had an automated defibrillator implantation for recurrent ventricular arrhythmias during pregnancy was referred to our third-level center at $24^{\text {th }}$ gestational week for setting up the follow-up and planning the delivery.

The lady was diagnosed in 2005 with LVNC by using echocardiographic and cardiac magnetic resonance imaging criteria $[3,4]$ (Figure1, panel B, C and D) and was treated with metoprolol (50 mg bid) for the treatment of isolated premature ventricular contractions. Family history was negative for cardiomyopathies and sudden cardiac death, and, at that time, the patient refused the genetic assessment after a psychological interview. In 2010 she 
had had an uneventful pregnancy and a normal ejection fraction (EF) was documented during hospitalisation. Three months later she started a new pregnancy, and at $15^{\text {th }}$ gestational week she was admitted to an another hospital for episodes of recurrent NSVT, symptomatic for pre-syncope (Figure 1, panel A). The echocardiographic evaluation confirmed the presence of a LVNC with a mild reduction of systolic function (EF 48\%). Blood tests were normal, while continuous cardiac monitoring revealed multiple episodes of NSVT. Due to severe episodes of hypotension metoprolol was replaced by sotalol (at the therapeutic dose of $240 \mathrm{mg} /$ day) but it was ineffective in reducing the number of ventricular events. Thus, considering the arrhythmic risk related to the type of cardiomyopathy, the presence of ventricular arrhythmias and assuming a probable worsening of the arrhythmic load with the increase of the volume overload in the following weeks, the implantation of an automated defibrillator (ICD) was decided. The clinical course was uneventful and the patient was discharged three days after the procedure while receiving beta-blocker therapy and low-molecular weight heparin.
She was then referred to our center and we performed monthly visits with echocardiographic evaluation and ICD interrogation. A crescendo of episodes of NSVT were recorded throughout the gestational course (Figure 2). The patient underwent an uncomplicated caesarean delivery at $38^{\text {th }}$ gestational week employing regional anesthesia. After delivery till to 6 months, a significant reduction of ventricular arrhythmic events was observed (Figure 2). In the following 5 years the patient remained asymptomatic (NYHA class I, LVEF 55\%) with no ICD interventions.

\section{Discussion}

LVNC can lead to life-threatening complications [5]. The clinical presentation of the disease is highly variable, and there is a paucity of data regarding pregnancy outcomes in women with LVNC characterised by an arrhythmic phenotype. The increased proarrhythmic burden could be the consequence of the major physiolog-
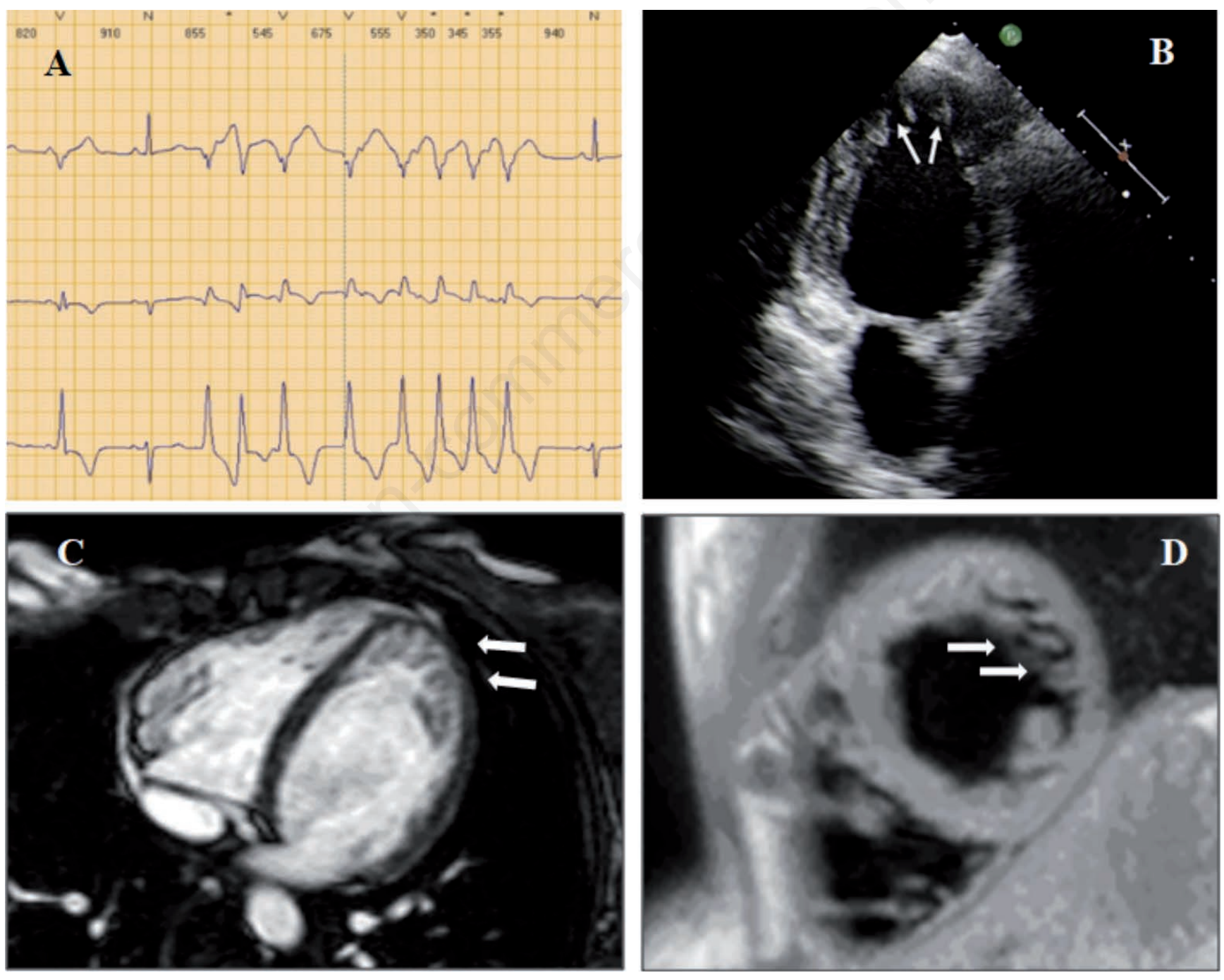

Figure 1. Non-sustained ventricular tachycardia during 24-hours continuous cardiac monitoring (panel A). Transthoracic echocardiogram from apical two-chamber view: evidence of prominent trabeculae and intratrabecular recesses (white arrows) (panel B). Cardiac Magnetic Resonance Imaging (MRI) on axial view (panel C) and in short axis view (T1-weighted) (panel D) showing the presence of trabeculae (white arrows) at the apex, in the infero-apical septum, and in the antero-apical portion of left ventricle. 


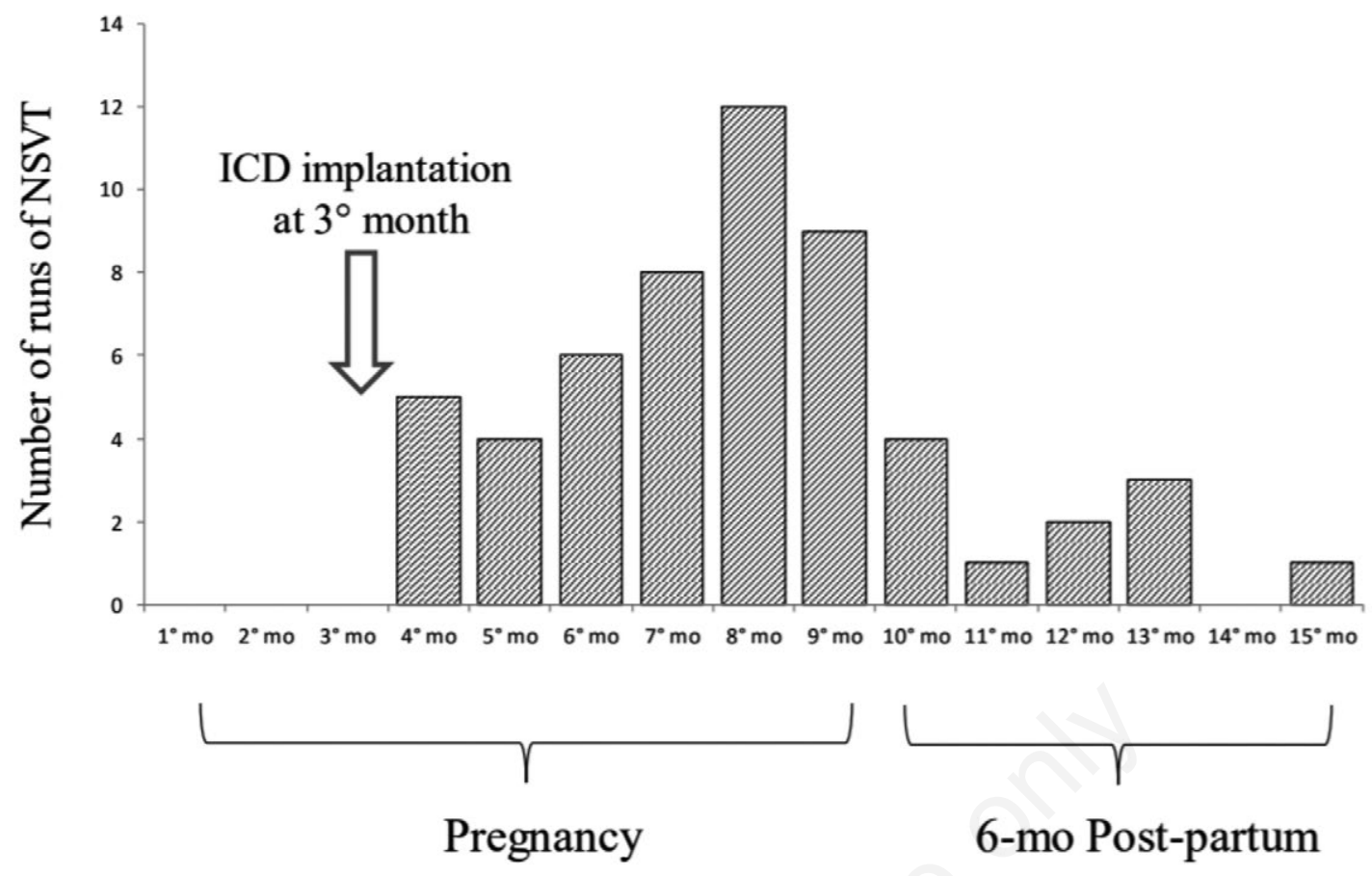

Figure 2. Monthly number of runs of Non-Sustained Ventricular Tachycardia (NSVT) during pregnancy and post-partum period at ICD interrogation. A crescendo of episodes of NSTV during gestation and a significant reduction after delivery is shown.

ical changes observed during pregnancy that could lead to hemodynamic compromise with deterioration of heart failure [6,7]. The long-term follow-up of our patient seems to suggest that the transient mild LV dysfunction was likely related to the interaction between the underlying cardiomyopathy and the two close pregnancies. On the other side the indication to implant an ICD for this type of presentation remains controversial as demonstrated by the absence of device intervention during pregnancy and at follow-up.

Therefore, a conservative attitude, with careful monitoring and antiarrhythmic prophylaxis therapy, could represent the most suitable choice considering that the arrhythmic load rapidly decreases after delivery. This case confirms the peculiarity of pregnancy condition and the real possible improvement of the clinical status after delivery. Nowadays in young patients such as pregnant women, when high-risk features are present, subcutaneous ICD could represent an effective alternative and should be considered for primary prevention especially in controversial situations.

\section{Conclusions}

The management of malignant arrhythmias during pregnancy is one of the concerns for patients with LVNC and requires a multidisciplinary approach in third-level centers that collect most of the cases and gain adequate experience in order not to underestimate or overestimate the cardiovascular risks of these patients.

\section{References}

1. Towbin JA, Jefferies JL. Cardiomyopathies Due to Left Ventricular Noncompaction, Mitochondrial and Storage Diseases, and Inborn Errors of Metabolism. Circ Res 2017;121:838-54.

2. Towbin JA, Lorts A, Jefferies JL. Left ventricular non-compaction cardiomyopathy. Lancet 2015;386:813-25.

3. Stöllberger C, Finsterer J. Left ventricular hypertrabeculation/noncompaction. J Am Soc Echocardiogr 2004;17:91-100.

4. Petersen SE, Selvanayagam JB, Wiesmann F et al. Left ventricular non-compaction: insights from cardiovascular magnetic resonance imaging, J Am Coll Cardiol 2005;46:101-5.

5. Hussein A, Karimianpour A, Collier P et al. Isolated noncompaction of the left ventricle in adults. JACC. 2015;66:578-85.

6. Krul SP, van der Smagt JJ, van den Berg MP et al. Systematic review of pregnancy in women with inherited cardiomyopathies. Eur J Heart Fail 2011;13:584-94.

7. Vaidya VR, Arora S, Patel N et al. Burden of Arrhythmia in Pregnancy. Circulation 2017;135:619-21. 